\title{
ON THE INTERSECTION OF FREE FACTORS OF A FREE GROUP
}

\author{
R. G. BURNS, T. C. CHAU AND D. SOLITAR
}

\begin{abstract}
An example is given of a descending sequence of free factors of a free group $F$ whose intersection is not a free factor of $F$.
\end{abstract}

It is known (see [1, Exercise 51(c), p. 49]) that the intersection of any set of direct summands of a countable free abelian group is again a direct summand. Verena Dyson has asked whether the corresponding statement is true for free factors of free groups. We give here a counterexample: there is in a free group $F$ of countably infinite rank a descending sequence of free factors whose intersection is not a free factor. (It is known that the intersection of a finite number of free factors of $F$ is again a free factor-this follows from the fact that if $K \leqslant A * B$ then $K \cap A$ is a free factor of $K$, which is in turn a consequence of the Kuroš subgroup theorem (see [2]). We mention also in passing that it is easy to find sequences of free factors of a free group whose union is not a free factor: Consider for example the subgroups of the free group on $x_{1}, x_{2}, \ldots$, generated by the finite subsets of $\left\{x_{1} x_{2}^{2}, x_{2} x_{3}^{2}\right.$, $\left.x_{3} x_{4}^{2}, \ldots\right\}$.)

We state our example as a theorem. We use the notation $[x, y]$ for the commutator $x^{-1} y^{-1} x y$.

THEOREM. Let $F$ be free on $\left\{a_{1}, a_{2}, \ldots ; x_{1}, x_{2}, \ldots\right\}$. Write

$$
H=\left\langle x_{1}\left[a_{1}, x_{2}\right], x_{2}\left[a_{2}, x_{3}\right], \ldots, x_{i}\left[a_{i}, x_{i+1}\right], \ldots\right\rangle,
$$

and for $i=1,2, \ldots$,

$$
B_{i}=\left\langle x_{1}\left[a_{1}, x_{2}\right], \ldots, x_{i}\left[a_{i}, x_{i+1}\right], x_{i+1}, x_{i+2}, \ldots ; a_{i+1}, a_{i+2}, \ldots\right\rangle .
$$

Then the $B_{i}$ are all free factors of $F, \cap_{i=1}^{\infty} B_{i}=H$, but $H$ is not a free factor of $F$.

Proof. It is easy to check that $B_{i} *\left\langle a_{1}, \ldots, a_{i}\right\rangle=F$, so that the $B_{i}$ are all free factors of $F$.

We next show that $H$ is not a free factor of $F$. Suppose the contrary; then the quotient of $F$ by the normal closure of $H$, say $G$, is free. In any case it has the presentation

$$
G=\left\langle a_{1}, a_{2}, \ldots ; x_{1}, x_{2}, \ldots \mid x_{1}=\left[x_{2}, a_{1}\right], \ldots, x_{i}=\left[x_{i+1}, a_{i}\right], \ldots\right\rangle .
$$

\section{Since}

Received by the editors December 23, 1976.

AMS (MOS) subject classifications (1970). Primary 20E05, $20 \mathrm{E} 30$. 


$$
x_{1}=\left[x_{2}, a_{1}\right]=\left[x_{3}, a_{2}, a_{1}\right]=\cdots=\left[x_{i}, a_{i-1}, \ldots, a_{1}\right]=\cdots,
$$

it follows that $x_{1}$ is in the intersection of the lower central series of $G$. Since $G$ is free this intersection is trivial, so that $x_{1}=1$ in $G$. We prove that this cannot be. To this end let $g_{1}$ be any nontrivial element of $A_{5}$, the alternating group on five letters. It is well known that every element of $A_{5}$ can be expressed as a commutator; thus $g_{1}=\left[g_{2}, h_{1}\right]$ for some $g_{2}, h_{1} \in A_{5}$. Define inductively $g_{i+1}, h_{i} \in A_{5}$ by choosing them arbitrarily subject to $g_{i}=\left[g_{i+1}, h_{i}\right]$. Then the map given by $x_{i} \rightarrow g_{i}, a_{i} \rightarrow h_{i}$, extends to a homomorphism from $G$ to $A_{5}$, which sends $x_{1}$ onto $g_{1} \neq 1$. Hence $G$ cannot be free and $H$ cannot be a free factor of $F$.

Finally we indicate why it is that $H=\cap B_{i}$. It is easy to verify that $\cap B_{i} \supseteq H$. For the reverse inclusion let $w$ be any element of $\cap B_{i}$. There exists an $n$ such that $w \in\left\langle a_{1}, \ldots, a_{n} ; x_{1}, \ldots, x_{n}\right\rangle$. If one now tries to express $w$ in terms of the free generators

$$
x_{1} a_{1}^{-1} x_{2}^{-1} a_{1} x_{2}, \ldots, x_{n} a_{n}^{-1} x_{n+1}^{-1} a_{n} x_{n+1}, x_{n+1}, x_{n+2}, \ldots ; a_{n+1}, a_{n+2}, \ldots,
$$

of $B_{n}$, then it is not difficult to see that $w$ can involve at most the first $n-1$ of these generators, so that $w \in H$ as required.

\section{REFERENCES}

1. Irving Kaplansky, Infinite abelian groups, Univ. of Michigan Press, Ann Arbor, 1954.

2. W. Magnus, A. Karrass and D. Solitar, Combinatorial group theory: Presentations of groups in terms of generators and relations, Pure and Appl. Math., vol. 13, Interscience, New York, 1966.

Department of Mathematics, York University, Downsview, Ontario, Canada

Department of Mathematics, Laurentian University, Sudbury, Ontario, Canada 